\title{
Resolving the $\Lambda$ Hypernuclear Overbinding Problem in Pionless Effective Field Theory
}

\author{
L. Contessi, ${ }^{1}$ N. Barnea, ${ }^{1}$ and A. Gal ${ }^{1, *}$ \\ ${ }^{1}$ Racah Institute of Physics, The Hebrew University, Jerusalem 91904, Israel
}

(Dated: September 11, 2018)

\begin{abstract}
We address the $\Lambda$-hypernuclear 'overbinding problem' in light hypernuclei which stands for a 1-3 MeV excessive $\Lambda$ separation energy calculated in ${ }_{\Lambda}^{5} \mathrm{He}$. This problem arises in most few-body calculations that reproduce ground-state $\Lambda$ separation energies in the lighter $\Lambda$ hypernuclei within various hyperon-nucleon interaction models. Recent pionless effective field theory ( $\$ \mathrm{EFT})$ nuclear few-body calculations are extended in this work to $\Lambda$ hypernuclei. At leading order, the $\Lambda N$ lowenergy constants are associated with $\Lambda N$ scattering lengths and the $\Lambda N N$ low-energy constants are fitted to $\Lambda$ separation energies $\left(B_{\Lambda}^{\exp }\right)$ for $A \leq 4$. The resulting $\not \mathrm{EFT}$ interaction reproduces in few-body stochastic variational method calculations the reported value $B_{\Lambda}^{\exp }\left({ }_{\Lambda}^{5} \mathrm{He}\right)=3.12 \pm 0.02 \mathrm{MeV}$ within a fraction of $\mathrm{MeV}$ over a broad range of $\not \mathrm{EFT}$ cut-off parameters. Possible consequences and extensions to heavier hypernuclei and to neutron-star matter are discussed.
\end{abstract}

PACS numbers:

Introduction. $\Lambda$ hypernuclei provide extension of atomic nuclei into the strangeness sector of hadronic matter 1]. Experimental data on $\Lambda$ hypernuclei are poorer, unfortunately, in both quantity and quality than the data available on normal nuclei. Nevertheless, the few dozen of $\Lambda$ separation energies $B_{\Lambda}^{\exp }$ determined across the periodic table, mostly for hypernuclear ground states, provide a useful test ground for the role of strangeness in dense hadronic matter, for example in neutron stars [2]. Particularly meaningful tests of hyperon-nucleon $(Y N)$ strong-interaction models are possible in light $\Lambda$ hypernuclei, $A \leq 5$, where precise few-body ab initio calculations are feasible [3].

TABLE I: Ground-state $\Lambda$ separation energies $B_{\Lambda}$ and excitation energies $E_{x}$ (in $\mathrm{MeV}$ ) from several few-body calculations of $s$-shell $\Lambda$ hypernuclei; see the text. Charge symmetry breaking (CSB) is included in the ${ }_{\Lambda}^{4} \mathrm{H}$ results from Ref. [11].

\begin{tabular}{ccccc}
\hline \hline & $B_{\Lambda}\left({ }_{\Lambda}^{3} \mathrm{H}\right)$ & $B_{\Lambda}\left({ }_{\Lambda}^{4} \mathrm{H}_{\mathrm{gs}}\right)$ & $E_{x}\left({ }_{\Lambda}^{4} \mathrm{H}_{\text {exc }}\right)$ & $B_{\Lambda}\left({ }_{\Lambda}^{5} \mathrm{He}\right)$ \\
Exp. & $0.13(5)[4]$ & $2.16(8)[5]$ & $1.09(2)[6]$ & $3.12(2)[4]$ \\
\hline DHT [7] & 0.10 & 2.24 & 0.36 & $\geq 5.16$ \\
AFDMCa & - & $1.97(11)[8]$ & - & $5.1(1)[9]$ \\
AFDMCb & $-1.2(2)[8]$ & $1.07(8)[8]$ & - & $3.22(14)[8]$ \\
$\chi$ EFTa & $0.11(1)[10]$ & $2.31(3)[11]$ & $0.95(15)[11]$ & $5.82(2)[12]$ \\
$\chi$ EFTb & - & $2.13(3)[11]$ & $1.39(15)[11]$ & $4.43(2)[12]$ \\
\hline \hline
\end{tabular}

The $\Lambda N$ interaction is not sufficiently strong to bind two-body systems. Hypernuclear binding starts with the weakly bound ${ }_{\Lambda}^{3} \mathrm{H}\left(I=0, J^{P}=\frac{1}{2}^{+}\right)$hypernucleus. No other $A=3$ hypernuclear level has ever been firmly established. The $A=4$ isodoublet hypernuclei $\left({ }_{\Lambda}^{4} \mathrm{H},{ }_{\Lambda}^{4} \mathrm{He}\right)$ each have two bound states, $0_{\mathrm{gs}}^{+}$and $1_{\mathrm{exc}}^{+}$. The hypernuclear $s$ shell ends with a single ${ }_{\Lambda}^{5} \mathrm{He}\left(I=0, J^{P}=\frac{1}{2}^{+}\right)$ level. Table \ demonstrates in chronological order the extent to which several representative few-body calculations overbind ${ }_{\Lambda}^{5} \mathrm{He}$ while reproducing the $B_{\Lambda}$ values of all other $s$-shell hypernuclear levels. This is known as the 'overbinding problem' in light $\Lambda$ hypernuclei since the
1972 work by Dalitz, Herndon and Tang (DHT) 7] who used a phenomenological $\Lambda N+\Lambda N N$ interaction model. The other, recent calculations listed in the table use the following methodologies:

(i) Auxiliary-field diffusion Monte Carlo (AFDMC) techniques within a $\Lambda N+\Lambda N N$ Urbana-type interaction model dating back to Bodmer, Usmani and Carlson [13]. Note that, while version AFDMCb 8] reproduces $B_{\Lambda}^{\exp }\left({ }_{\Lambda}^{5} \mathrm{He}\right)$ as a prerequisite to resolving the 'hyperon puzzle' in neutron-star matter [2], it underbinds the lighter $s$-shell hypernuclei by about $1 \mathrm{MeV}$ each and, thus, does not resolve the overbinding problem as defined here. A revision of this work [14 suggests that by modifying some of the $\Lambda N N$ strength parameters it is possible to avoid the underbinding.

(ii) No-core shell-model techniques within a leading-order (LO) chiral effective field theory ( $\chi \mathrm{EFT}) Y N$ interaction model, with momentum cut-off values of 600 (a) and 700 (b) $\mathrm{MeV} / \mathrm{c}$, in which three-body $\Lambda N N$ terms are induced through $\Lambda N \leftrightarrow \Sigma N$ coupling. The ${ }_{\Lambda}^{5} \mathrm{He} \chi \mathrm{EFT}$ results listed here were obtained by employing a similarity renormalization group transformation [12], reducing the model-space dimension in order to enhance the poor convergence met in using bare $Y N$ interactions [15]. No $\chi$ EFT calculations have been reported yet for ${ }_{\Lambda}^{5} \mathrm{He}$ at next-to-leading order (NLO).

Excluding calculations using an uncontrolled number of interaction terms, the only published few-body calculations claiming to have solved the overbinding problem are those by Nemura et al. [16]. However, it was realized by Nogga, Kamada and Glöckle [17] that a more faithful reproduction of the Nijmegen soft-core (NSC) meson-exchange potentials used in these calculations in fact underbinds appreciably the $A=4$ hypernuclei. Thus, the overbinding problem is still alive and kicking, with ${ }_{\Lambda}^{5} \mathrm{He}$ overbound by $1-3 \mathrm{MeV}$ in the recent few-body calculations listed in Table \

The present work reports on few-body stochastic vari- 
ational method (SVM) precise calculations of $s$-shell hypernuclei, using Hamiltonians constructed at LO in a pionless effective field theory ( $九 \mathrm{EFT}$ ) approach. This is accomplished by extending a purely nuclear $九$ EFT Hamiltonian used in few-nucleon calculations, first reported in Refs. [18, 19 and more recently also in lattice-nuclei calculations [20 23], to include $\Lambda$ hyperons. With $\Lambda N$ one-pion exchange (OPE) forbidden by isospin invariance, the $\pi$ EFT breakup scale is $2 m_{\pi}$, remarkably close to the threshold value $p_{\Lambda N}^{\text {th }} \approx 283 \mathrm{MeV} / \mathrm{c}$ for exciting $\Sigma N$ pairs in $\pi$ EFT approaches 24]. A typical momentum scale $Q$ in ${ }_{\Lambda}^{5} \mathrm{He}$ is $p_{\Lambda} \approx \sqrt{2 M_{\Lambda} B_{\Lambda}}=83 \mathrm{MeV} / \mathrm{c}$, suggesting a $\not$ EFT expansion parameter $\left(Q / 2 m_{\pi}\right) \approx 0.3$ for $s$-shell hypernuclei. This implies a $\not$ EFT LO accuracy of the order of $\left(Q / 2 m_{\pi}\right)^{2} \approx 9 \%$. A somewhat larger value is obtained by using a mean $\Lambda N$ pair breakup energy in ${ }_{\Lambda}^{5} \mathrm{He}, B_{\Lambda N}=\left(B_{\Lambda}+B_{N}\right) / 2=12.1 \mathrm{MeV}$, to estimate $p_{\Lambda N} \approx \sqrt{2 \mu_{\Lambda N} B_{\Lambda N}}$ in light $\Lambda$ hypernuclei. This yields $p_{\Lambda N} \approx 111 \mathrm{MeV} / \mathrm{c}$ and $\left(p_{\Lambda N} / 2 m_{\pi}\right)^{2} \approx 0.16$. With past tEFT $\Lambda$ hypernuclear applications limited to $A=3$ systems [25, 26], ours is the first comprehensive application of $\pitchfork \mathrm{EFT}$ to the full hypernuclear $s$ shell.

As shown in this Letter, our few-body SVM calculations of light $\Lambda$ hypernuclei in the $₫ \mathrm{EFT}$ approach largely resolve the overbinding problem for ${ }_{\Lambda}^{5} \mathrm{He}$ to the accuracy expected at LO. Below, we expand briefly on the EFF approach, its input, and the SVM few-body calculations applied in the present work to light nuclei and hypernuclei. Possible consequences of resolving the hypernuclear overbinding problem in light hypernuclei and extensions to heavier systems are discussed in the concluding paragraphs.

Application of $\not \mathrm{EFT}$ to $\Lambda$ hypernuclei. Hadronic systems consisting of neutrons, protons, and $\Lambda$-hyperons are described in $\not \mathrm{EFT}$ by a Lagrangian density

$\mathcal{L}=N^{\dagger}\left(i \partial_{0}+\frac{\nabla^{2}}{2 M_{N}}\right) N+\Lambda^{\dagger}\left(i \partial_{0}+\frac{\nabla^{2}}{2 M_{\Lambda}}\right) \Lambda+\mathcal{L}_{2 B}+\mathcal{L}_{3 B}+\ldots$,

where $N$ and $\Lambda$ are nucleon and $\Lambda$-hyperon fields, respectively, and $\mathcal{L}_{2 B}, \mathcal{L}_{3 B}, \ldots$ are two-body, three-body, and, in general, $n$-body interaction terms. The interaction terms are composed of $N, \Lambda$ fields and their derivatives subject to symmetry constraints that $\mathcal{L}$ is scalar and isoscalar and to a power counting that orders them according to their importance. At LO, the Lagrangian contains only contact two-body and three-body $s$-wave interaction terms; i.e., $\mathcal{L}_{2 B}$ and $\mathcal{L}_{3 B}$ are the sum of all possible $N, \Lambda$ field combinations, with no derivatives, that create an $s$-wave projection operator. Thus, there is a one-to-one correspondence between LO interaction terms and all possible $N N, N \Lambda, \Lambda \Lambda$ and $N N N, N N \Lambda, \ldots$ $s$-wave states. Each of these terms is associated with its own low-energy constant (LEC). In the present work we focus on single- $\Lambda$ hypernuclei and, hence, ignore all terms in $\mathcal{L}$ containing more than one $\Lambda_{\alpha}^{\dagger} \Lambda_{\beta}$ field pair.
Momentum-dependent interaction terms, such as tensor or spin-orbit, appear at subleading order in $\not \mathrm{EFT}$ power counting [18]. In particular, the long-range $\Lambda N$ tensor force induced by a $\Lambda N \rightarrow \Sigma N$ OPE transition followed by a $\Sigma N \rightarrow \Lambda N$ OPE transition is expected to be weak because this two-pion exchange mechanism is dominated by its central $S \rightarrow D \rightarrow S$ component, which is partially absorbed in the $\Lambda N$ and $\Lambda N N$ LO contact LECs. Short-range $K$ and $K^{*}$ exchanges add a rather weak direct $\Lambda N$ tensor force [27, 28], as also deduced from several observed $p$-shell $\Lambda$ hypernuclear spectra [29].

The contact interactions of the Lagrangian $\mathcal{L}$ are regularized by introducing a local Gaussian regulator with momentum cut-off $\lambda$ (see, e.g., 30]):

$$
\delta_{\lambda}(\boldsymbol{r})=\left(\frac{\lambda}{2 \sqrt{\pi}}\right)^{3} \exp \left(-\frac{\lambda^{2}}{4} \boldsymbol{r}^{2}\right)
$$

that smears contact terms over distances $\sim \lambda^{-1}$, becoming a Dirac $\delta^{(3)}(\boldsymbol{r})$ in the limit $\lambda \rightarrow \infty$. The cut-off parameter $\lambda$ may be viewed as a scale parameter with respect to typical values of momenta $Q$. To make observables independent of specific values of $\lambda$, the LECs must be properly renormalized. Truncating $₫ \mathrm{EFT}$ at LO and using values of $\lambda$ higher than the breakup scale of the theory (here $\approx 2 m_{\pi}$ ), observables acquire a residual dependence $O(Q / \lambda)$ which diminishes with increasing $\lambda$.

TABLE II: Input scattering lengths (in fm) used to fit tEFT two-body LECs; see the text.

\begin{tabular}{cccccc}
\hline \hline$Y N$ model & Ref. & $a_{s}(N N)$ & $a_{s}(\Lambda N)$ & $a_{t}(\Lambda N)$ & $\bar{a}_{\Lambda N}$ \\
\hline Alexander[A] & {$[31]$} & -23.72 & -1.8 & -1.6 & -1.65 \\
Alexander[B] & {$[31]$} & -18.63 & -1.8 & -1.6 & -1.65 \\
NSC97f & {$[32]$} & -18.63 & -2.60 & -1.71 & -1.93 \\
$\chi$ EFT(LO) & {$[33]$} & -18.63 & -1.91 & -1.23 & -1.40 \\
$\chi$ EFT(NLO) & {$[34]$} & -18.63 & -2.91 & -1.54 & -1.88 \\
\hline \hline
\end{tabular}

The resulting LO two-body interaction is given by

$$
V_{2 B}=\sum_{I S} C_{\lambda}^{I S} \sum_{i<j} \mathcal{P}_{I S}(i j) \delta_{\lambda}\left(\boldsymbol{r}_{i j}\right),
$$

where $\mathcal{P}_{I S}$ are projection operators on $N N, \Lambda N$ pairs with isospin $I$ and spin $S$ and $C_{\lambda}^{I S}$ are LECs, fixed by fitting to low-energy two-body observables, e.g., to the corresponding $N N$ and $\Lambda N$ scattering lengths. In the present work the $N N I S=01$ LEC is fitted to the deuteron binding energy, hardly affecting the results obtained alternatively by fitting to the $I S=01$ scattering length. The scattering lengths used to fit the LECs are listed in Table II For $I S=10$, two choices of a chargeindependent $N N$ spin-singlet scattering length, $[\mathrm{A}]$ and [B], were made for comparison [35]. For $\Lambda N$ scattering lengths we used best-fit values derived from the lowenergy $\Lambda p$ spin-averaged scattering cross sections measured by Alexander et al. 31], assuming charge sym- 
metry, and also values from several listed $Y N$ interaction models. These choices suggest a ${ }^{1} S_{0} \Lambda N$ interaction stronger than in ${ }^{3} S_{1}$, spanning a broad range of possible $\Lambda N$ spin dependence. Also listed are values of the spin-averaged $\Lambda N$ scattering length $\bar{a}=\left(3 a_{t}+a_{s}\right) / 4$, with approximately $\pm 16 \%$ spread about the best-fit value $-1.65 \mathrm{fm}$ from Ref. 31], reflecting the model dependence of fitting all low-energy $Y N$ scattering and reaction cross section data [36.

The LO three-body interaction consists of a single $N N N$ term associated with the $I S=\frac{1}{2} \frac{1}{2}$ channel and of three $\Lambda N N$ terms associated with the $I S=0 \frac{1}{2}, 1 \frac{1}{2}, 0 \frac{3}{2}$ $s$-wave configurations. The explicit form of the threebody $N N N$ potential is given by

$$
V_{N N N}=D_{\lambda}^{\frac{1}{2} \frac{1}{2}} \sum_{i<j<k} \mathcal{Q}_{\frac{1}{2} \frac{1}{2}}(i j k)\left(\sum_{c y c} \delta_{\lambda}\left(\boldsymbol{r}_{i k}\right) \delta_{\lambda}\left(\boldsymbol{r}_{j k}\right)\right),
$$

where the first sum runs over all $N N N$ triplets. The three-body $\Lambda N N$ potential is given by

$$
V_{\Lambda N N}=\sum_{I S} D_{\lambda}^{I S} \sum_{i<j} \mathcal{Q}_{I S}(i j \Lambda) \delta_{\lambda}\left(\boldsymbol{r}_{i \Lambda}\right) \delta_{\lambda}\left(\boldsymbol{r}_{j \Lambda}\right),
$$

where the second sum runs over all $N N$ pairs. In Eqs. (4) and (5), $\mathcal{Q}_{I S}$ are projection operators on baryon triplets with isospin $I$ and spin $S$, and $D_{\lambda}^{I S}$ are LECs.

There are four three-body LECs, a pure $N N N$ LEC $D_{\lambda}^{\frac{1}{2} \frac{1}{2}}$ fitted to $B\left({ }^{3} \mathrm{H}\right)$ and three $\Lambda N N$ LECs associated with the three possible $s$-wave $\Lambda N N$ systems. Because only ${ }_{\Lambda}^{3} \mathrm{H}\left(I=0, J^{P}=\frac{1}{2}^{+}\right)$is known to be bound, we have fitted these LECs instead to the three $B_{\Lambda}$ values available (disregarding CSB) for $A \leq 4:{ }_{\Lambda}^{3} \mathrm{H}\left(I=0, J^{P}=\frac{1}{2}^{+}\right.$) for $D_{\lambda}^{0 \frac{1}{2}},{ }_{\Lambda}^{4} \mathrm{H}_{\mathrm{gs}}\left(I=\frac{1}{2}, J^{P}=0^{+}\right)$subsequently for $D_{\lambda}^{1 \frac{1}{2}}$, and finally ${ }_{\Lambda}^{4} \mathrm{H}_{\text {exc }}\left(I=\frac{1}{2}, J^{P}=1^{+}\right)$for $D_{\lambda}^{0 \frac{3}{2}}$. Altogether, eight LECs at LO are constrained by few-body nuclear and hypernuclear data, to be subsequently used in calculations of ${ }^{4} \mathrm{He}$ and ${ }_{\Lambda}^{5} \mathrm{He}$.

Stochastic variational method (SVM). To solve the $A$-body Schrödinger equation, the wave function $\Psi$ is expanded on a correlated Gaussian basis. Introducing a vector $\boldsymbol{x}=\left(\boldsymbol{x}_{1}, \boldsymbol{x}_{2}, \ldots, \boldsymbol{x}_{A-1}\right)$ of Jacobi vectors $\boldsymbol{x}_{j}$, $j=1,2, \ldots, A-1$, we may write $\Psi$ as

$$
\Psi=\sum_{k} c_{k} \hat{\mathcal{A}}\left\{\chi_{S M}^{k} \xi_{I_{z}}^{k} \exp \left(-\frac{1}{2} \boldsymbol{x}^{T} A_{k} \boldsymbol{x}\right)\right\},
$$

where the operator $\hat{\mathcal{A}}$ antisymmetrizes over nucleons. In Eq. (6) the basis states are defined by the real, symmetric and positive-definite $(A-1) \times(A-1)$ matrix $A_{k}$, together with the spin and isospin functions $\chi_{S}$ and $\xi_{I}$. Once these are chosen, the linear variational parameters $c_{k}$ are obtained through diagonalization of the Hamiltonian matrix. The matrix $A_{k}$ introduces $A(A-1) / 2$ nonlinear variational parameters which are chosen stochastically, hence the name SVM. For a comprehensive review, see Ref. [37]. For the specific calculation of the three-body interaction matrix elements, see Ref. [30].

Results and discussion. The $\not \mathrm{EFT}$ approach with two-body and three-body regulated contact terms defined by Eqs. (3) - (5) was applied in SVM few-body calculations as outlined above to the $s$-shell nuclei and hypernuclei using the $\Lambda N$ scattering-length combinations listed in Table II The calculated ${ }_{\Lambda}^{5} \mathrm{He}$ binding energy $B\left({ }_{\Lambda}^{5} \mathrm{He}\right)$ along with $B\left({ }^{4} \mathrm{He}\right)$ are found to depend only moderately on $\lambda$, for $\lambda \gtrsim 2 \mathrm{fm}^{-1}$, exhibiting renormalization scale invariance in the limit $\lambda \rightarrow \infty$. Using $a_{s}(N N)=-18.63 \mathrm{fm}$, we obtain in this limit $B\left({ }^{4} \mathrm{He}\right) \rightarrow 29.2 \pm 0.5 \mathrm{MeV}$, which compares well with $B_{\exp }\left({ }^{4} \mathrm{He}\right)=28.3 \mathrm{MeV}$, given that our tEFT is truncated at $\mathrm{LO}$ and considering that the suppressed Coulomb force is expected to reduce $B\left({ }^{4} \mathrm{He}\right)$ further by roughly $1 \mathrm{MeV}$. The binding energies $B\left({ }^{4} \mathrm{He}\right)$ calculated for the other choice, $a_{s}(N N)=-23.72 \mathrm{fm}$, differ by less than $0.4 \mathrm{MeV}$ and agree with those calculated recently in Ref. 22].

With $B\left({ }^{4} \mathrm{He}\right)$ and $B\left({ }_{\Lambda}^{5} \mathrm{He}\right)$ computed, we show in Fig. 11 the resulting $\Lambda$ separation energy values $B_{\Lambda}\left({ }_{\Lambda}^{5} \mathrm{He}\right)$ as a function of the cut-off $\lambda$ for the $\Lambda N$ scattering-length versions Alexander[B] and $\chi \mathrm{NLO}$ listed in Table [II The results shown for Alexander[B] agree to a level of $1 \%$ with those (not shown) for Alexander[A]; both versions differ only in their ${ }^{1} S_{0} N N$ input. The dependence of the calculated $B_{\Lambda}\left({ }_{\Lambda}^{5} \mathrm{He}\right)$ values on $\lambda$ is similar in all versions, switching from about $2-3 \mathrm{MeV}$ overbinding at $\lambda=1 \mathrm{fm}^{-1}$ to less than $1 \mathrm{MeV}$ underbinding between $\lambda=2$ and $3 \mathrm{fm}^{-1}$, and smoothly varying beyond, approaching a finite limit at $\lambda \rightarrow \infty$. Renormalization scale invariance implies that $B_{\Lambda}\left({ }_{\Lambda}^{5} \mathrm{He}\right)$ should be considered in this limit. However, it may be argued that, when the cut-off value $\lambda$ matches the EFT breakup scale, higher-order terms such as effective-range corrections are absorbed into the LECs. A reasonable choice of finite cut-off values in the present case is between $\lambda \approx 1.5 \mathrm{fm}^{-1}$, which marks the \#EFT breakup scale of $2 m_{\pi}$, and $4 \mathrm{fm}^{-1}$, beginning at which the detailed dynamics of vector-meson exchanges may require attention. In the following we compare the finite versus infinite options for $\lambda$.

TABLE III: $B_{\Lambda}\left({ }_{\Lambda}^{5} \mathrm{He}\right)$ values $(\mathrm{MeV})$ in $\mathrm{LO} \not$ EFT calculations for several $\Lambda N$ scattering-length versions from Table II. The uncertainties listed for cut-off $\lambda=4 \mathrm{fm}^{-1}$ are due to subtracting $B\left({ }^{4} \mathrm{He}\right)$ from $B\left({ }_{\Lambda}^{5} \mathrm{He}\right)$, whereas those for $\lambda \rightarrow \infty$ are mostly from extrapolation, with fitting uncertainties $\lesssim 10 \mathrm{keV}$.

\begin{tabular}{ccccc}
\hline \hline$\lambda\left(\mathrm{fm}^{-1}\right)$ & Alexander[B] & NSC97f & $\chi \mathrm{LO}$ & $\chi \mathrm{NLO}$ \\
\hline 4 & $2.59(3)$ & $2.32(3)$ & $2.99(3)$ & $2.40(3)$ \\
$\rightarrow \infty$ & $3.01(10)$ & $2.74(11)$ & $3.96(08)$ & $3.01(06)$ \\
\hline \hline
\end{tabular}

Calculated values of $B_{\Lambda}\left({ }_{\Lambda}^{5} \mathrm{He}\right)$ are listed in Table $\llbracket$ for $\lambda=4 \mathrm{fm}^{-1}$ and as extrapolated to $\lambda \rightarrow \infty$. To extrapolate to $\lambda \rightarrow \infty$, the calculated $B(\lambda)$ values can be fitted by a 

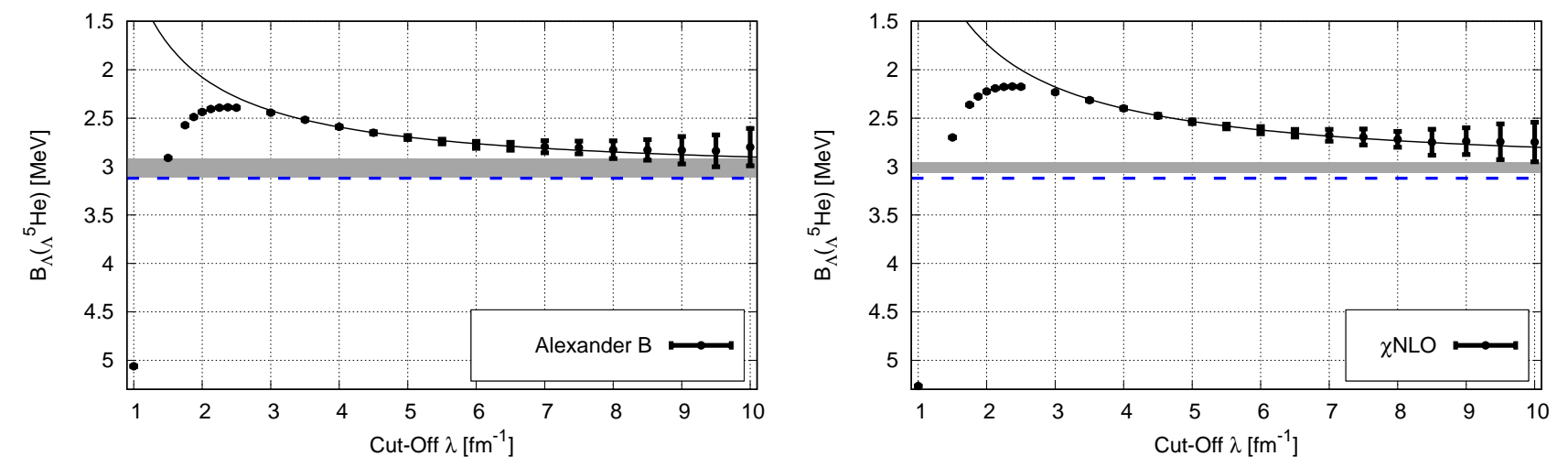

FIG. 1: $B_{\Lambda}\left({ }_{\Lambda}^{5} \mathrm{He}\right)(\mathrm{MeV})$ as a function of the cut-off $\lambda\left(\mathrm{fm}^{-1}\right)$ in LO $\not$ EFT calculations with $\Lambda N$ scattering-length input listed in Table II Solid lines mark a two-parameter fit $a+b / \lambda$, starting from $\lambda=4 \mathrm{fm}^{-1}$. Gray horizontal bands mark $\lambda \rightarrow \infty$ extrapolation uncertainties. Dashed horizontal lines mark the value $B_{\Lambda}^{\exp }\left({ }_{\Lambda}^{5} \mathrm{He}\right)=3.12 \pm 0.02 \mathrm{MeV}$.

power series in the small parameter $Q / \lambda$ :

$$
\frac{B(\lambda)}{B(\infty)}=\left[1+\alpha \frac{Q}{\lambda}+\beta\left(\frac{Q}{\lambda}\right)^{2}+\gamma\left(\frac{Q}{\lambda}\right)^{3}+\ldots\right] .
$$

The extrapolation uncertainties listed in Table $\amalg$ for the asymptotic values $B_{\Lambda}(\lambda \rightarrow \infty)$ were derived by comparing two- and three-parameter fits of this form. These uncertainties are also shown as gray bands in Fig. 1 above. The table demonstrates how $\Lambda N$ version $\chi \mathrm{LO}$, of all versions, is close to reproducing $B_{\Lambda}^{\exp }\left({ }_{\Lambda}^{5} \mathrm{He}\right)$ for $\lambda=4 \mathrm{fm}^{-1}$, whereas versions Alexander[B] and $\chi \mathrm{NLO}$ (see also Fig. 1) do so only in the limit $\lambda \rightarrow \infty$.

The sign and size of the three-body contributions play a crucial role in understanding the cut-off $\lambda$ dependence of the calculated $B_{\Lambda}\left({ }_{\Lambda}^{5} \mathrm{He}\right)$. The nuclear $N N N$ term first changes from weak attraction at $\lambda=1 \mathrm{fm}^{-1}$ in ${ }^{3} \mathrm{H}$ and ${ }^{4} \mathrm{He}$, similar to that required in phenomenological models [38], to strong repulsion at $\lambda=2 \mathrm{fm}^{-1}$, which reaches maximal values around $\lambda=4 \mathrm{fm}^{-1}$. However, for larger values of $\lambda$ it decreases slowly. The $\Lambda N N$ contribution follows a similar trend, but it is weaker than the $N N N$ contribution by a factor of roughly 3 when repulsive. The transition of the three-body contributions from longrange weak attraction to relatively strong repulsion for short-range interactions is correlated with the transition seen in Fig. 1 from strongly overbinding ${ }_{\Lambda}^{5}$ He to weakly underbinding it. We note that for $\lambda \gtrsim 1.5 \mathrm{fm}^{-1}$ all of the three $\Lambda N N$ components are repulsive, as required to avoid Thomas collapse, imposing thereby some constraints on the $\Lambda N N$ LECs.

Finally, using the $\not$ EFT LECs derived here to evaluate $B_{\Lambda}$ in symmetric nuclear matter (SNM), we have found within a simple Fermi gas model that for version Alexander[B], for example, $B_{\Lambda}(\mathrm{SNM}) \leq 27 \mathrm{MeV}$ at nuclear saturation density, $\rho_{A}=0.16 \mathrm{fm}^{-3}$, for any cut-off value $\lambda$. Although this value is only a lower bound on the binding energy of $\Lambda$ in SNM, the acceptable value being $\approx 30 \mathrm{MeV}$ [1, it is encouraging that our $\not \mathrm{EFT}$ does not lead to excessive binding. This calls for more rigorous evaluations of $B_{\Lambda}(\mathrm{SNM})$ using perhaps advanced Monte Carlo variational techniques.

Summary and outlook. The present work was motivated by the $1-3 \mathrm{MeV}$ persistent overbinding of ${ }_{\Lambda}^{5} \mathrm{He}$ in most of the few-body calculations reported to date, including recent LO EFT model calculations [12]. To this end, we have applied the $\not \mathrm{EFT}$ approach at LO to $s$-shell $\Lambda$ hypernuclei within precise few-body SVM calculations, extending recent $\not \mathrm{EFT}$ studies of light nuclei 20 23]. This required five LECs at LO: two $\Lambda N$ LECs, related here to spin-triplet and spin-singlet $\Lambda N$ scattering lengths in several $\Lambda N$ interaction models, and three $\Lambda N N$ LECs fitted to the three available $B_{\Lambda}$ values in the $A=3,4$ hypernuclei. With these five fitted LECs, for each of the momentum scale parameters $\lambda$ chosen, the $\Lambda$ separation energy $B_{\Lambda}\left({ }_{\Lambda}^{5} \mathrm{He}\right)$ was evaluated. Our main finding is that, while ${ }_{\Lambda}^{5} \mathrm{He}$ is overbound indeed by up to $3 \mathrm{MeV}$ for relatively long-range $\Lambda N$ and $\Lambda N N$ interactions, say at $\lambda \sim 1 \mathrm{fm}^{-1}$, it quickly becomes underbound by less than $1 \mathrm{MeV}$ for $\lambda \sim 2-3 \mathrm{fm}^{-1}$. For most of the $\Lambda N$ scattering-length versions studied here, $B_{\Lambda}^{\text {calc }}\left({ }_{\Lambda}^{5} \mathrm{He}\right)$ approaches slowly in the limit $\lambda \rightarrow \infty$ the value $B_{\Lambda}^{\exp }\left({ }_{\Lambda}^{5} \mathrm{He}\right)=3.12 \pm 0.02 \mathrm{MeV}$, notably for version Alexander $[\mathrm{B}]$ derived in a model independent way directly from experiment.

Having largely resolved the overbinding problem in light $\Lambda$ hypernuclei, it would be interesting in future work to study possible implications of the strong three-body $\Lambda N N$ interactions found here to other problems that involve hyperons in nuclear and neutron-star matter. To be more specific, we make the following observations:

(i) Other than the $s$-shell hypernuclei studied in the present work, $p$-shell hypernuclei offer a well-studied range of mass numbers $6 \leq A \leq 16$ both experimentally and theoretically [1]. Recent $\chi$ EFT LO calculations [39] 
using induced $Y N N$ repulsive contributions suggest that the $s$-shell overbinding problem extends to the $p$ shell. In contrast, shell-model studies [29] reproduce satisfactorily $p$-shell ground-state $B_{\Lambda}$ values, essentially by using $B_{\Lambda}^{\exp }\left({ }_{\Lambda}^{5} \mathrm{He}\right)$ for input, except for the relatively large difference of about $1.8 \mathrm{MeV}$ between $B_{\Lambda}\left({ }_{\Lambda}^{9} \mathrm{Li}\right)$ and $B_{\Lambda}\left({ }_{\Lambda}^{9} \mathrm{Be}\right)$. In fact, it was noted long ago that strongly repulsive $\Lambda N N$ terms could settle it [40]. It would be interesting to apply our derived $\Lambda N N$ interaction terms in future shell-model calculations.

(ii) The $\not$ EFT Hamiltonian derived here includes already at LO repulsive $\Lambda N N$ terms which are qualitatively as strong as those used by Lonardoni, Pederiva and Gandolfi [8] to resolve the hyperon puzzle [2]. It would be interesting then to apply our $\Lambda N+\Lambda N N$ interaction terms in state-of-the-art neutron-star matter calculations to see whether or not their suggested resolution of the hyperon puzzle is sufficiently robust.

We hope to discuss in greater detail some of these issues in forthcoming studies.

The work of L.C. and N.B. was supported by the Pazy Foundation and by the Israel Science Foundation Grant No. 1308/16.

* avragal@savion.huji.ac.il

[1] A. Gal, E.V. Hungerford, and D.J. Millener, Rev. Mod. Phys. 88, 035004 (2016).

[2] D. Lonardoni, A. Lovato, S. Gandolfi, and F. Pederiva, Phys. Rev. Lett. 114, 092301 (2015).

[3] A. Nogga, Nucl. Phys. A 914, 140 (2013).

[4] D.H. Davis, Nucl. Phys. A 754, 3c (2005).

[5] A. Esser et al. (MAMI A1 Collaboration), Phys. Rev. Lett. 114, 232501 (2015); F. Schulz et al. (MAMI A1 Collaboration), Nucl. Phys. A 954, 149 (2016).

[6] T.O. Yamamoto et al. (J-PARC E13 Collaboration), Phys. Rev. Lett. 115, 222501 (2015).

[7] R.H. Dalitz, R.C. Herndon, and Y.C. Tang, Nucl. Phys. B 47, 109 (1972).

[8] D. Lonardoni, F. Pederiva, and S. Gandolfi, Phys. Rev. C 89, 014314 (2014).

[9] D. Lonardoni, S. Gandolfi, and F. Pederiva, Phys. Rev. C 87, 041303(R) (2013).

[10] R. Wirth, D. Gazda, P. Navrátil, A. Calci, J. Langhammer, and R. Roth, Phys. Rev. Lett. 113, 192502 (2014).

[11] D. Gazda and A. Gal, Phys. Rev. Lett. 116, 122501 (2016); Nucl. Phys. A 954, 161 (2016).

[12] R. Wirth and R. Roth, Phys. Lett. B 779, 336 (2018). We thank Roland Wirth for providing us with these values.

[13] A.R. Bodmer, Q.N. Usmani, and J. Carlson, Phys. Rev. C 29, 684 (1984).
[14] D. Lonardoni and F. Pederiva, arXiv:1711.07521 and private communication (June 2018).

[15] R. Wirth, D. Gazda, P. Navrátil, and R. Roth, Phys. Rev. C 97, 064315 (2018).

[16] H. Nemura, Y. Akaishi, and Y. Suzuki, Phys. Rev. Lett. 89, 142504 (2002); see also Y. Akaishi, T. Harada, S. Shinmura, and K.S. Myint, Phys. Rev. Lett. 84, 3539 (2000).

[17] A. Nogga, H. Kamada, and W. Glöckle, Phys. Rev. Lett. 88, 172501 (2002).

[18] U. van Kolck, Nucl. Phys. A 645, 273 (1999).

[19] P.F. Bedaque, H.-W. Hammer, and U. van Kolck, Nucl. Phys. A 676, 357 (2000).

[20] N. Barnea, L. Contessi, D. Gazit, F. Pederiva, and U. van Kolck, Phys. Rev. Lett. 114, 052501 (2015).

[21] J. Kirscher, N. Barnea, D. Gazit, F. Pederiva, and U. van Kolck, Phys. Rev. C 92, 054002 (2015).

[22] L. Contessi, A. Lovato, F. Pederiva, A. Roggero, J. Kirscher, and U. van Kolck, Phys. Lett. B 772, 839 (2017).

[23] J. Kirscher, E. Pazy, J. Drachman, and N. Barnea, Phys. Rev. C 96, 024001 (2017).

[24] J. Haidenbauer, U.-G. Meißner, A. Nogga, and H. Polinder, Lect. Notes Phys. 724, 113 (2007).

[25] H.-W. Hammer, Nucl. Phys. A 705, 173 (2002).

[26] S.-I. Ando, U. Raha, and Y. Oh, Phys. Rev. C 92, 024325 (2015).

[27] A. Gal, J.M. Soper, and R.H. Dalitz, Ann. Phys. (N.Y.) 63, 53 (1971); see in particular Eq. (5.2) therein.

[28] D.J. Millener, A. Gal, C.B. Dover, and R.H. Dalitz, Phys. Rev. C 31, 499 (1985).

[29] D.J. Millener, Nucl. Phys. A 881, 298 (2012).

[30] B. Bazak, M. Eliyahu, and U. van Kolck, Phys. Rev. A 94, 052502 (2016).

[31] G. Alexander, U. Karshon, A. Shapira, et al., Phys. Rev. 173, 1452 (1968).

[32] Th.A. Rijken, V.G.J. Stoks, and Y. Yamamoto, Phys. Rev. C 59, 21 (1999).

[33] H. Polinder, J. Haidenbauer, and U.-G. Meißner, Nucl. Phys. A 779, 244 (2006).

[34] J. Haidenbauer, S. Petschauer, N. Kaiser, U.-G. Meißner, A. Nogga, and W. Weise, Nucl. Phys. A 915, 24 (2013).

[35] These values agree within uncertainties with accepted values $a_{s}^{p n}=-23.77 \pm 0.09$ and $a_{s}^{n n}=-18.8 \pm 0.3 \mathrm{fm}$, see G.A. Miller, A.K. Opper, and E.J. Stephenson, Annu. Rev. Nucl. Part. Sci. 56, 253 (2006).

[36] To demonstrate such model dependence, see, e.g., Fig. 5(a) in Ref. 24].

[37] Y. Suzuki and K. Varga, Stochastic Variational Approach to Quantum Mechanical Few-Body Problems (SpringerVerlag, Berlin, 1998).

[38] A. Nogga, H. Kamada, and W. Glöckle, Phys. Rev. Lett. 85, 944 (2000).

[39] R. Wirth and R. Roth, Phys. Rev. Lett. 117, 182501 (2016).

[40] A. Gal, Phys. Rev. Lett. 18, 568 (1967). 